\title{
Application of Cu-FAU nanozeolites for decontamination of surfaces soiled with the ESKAPE pathogens
}

James Redfern, ${ }^{\mathrm{a}}$ Kamila Goldyn, ${ }^{\mathrm{b}}$ Joanna Verran, ${ }^{\mathrm{a}}$ Richard Retoux, ${ }^{\mathrm{c}}$ Lubomira Tosheva, ,a Svetlana Mintova, ${ }^{*}$

${ }^{\text {a }}$ Faculty of Science and Engineering, Manchester Metropolitan University, Chester Street, Manchester, M1 5GD, United Kingdom

b LCS, CNRS, ENSICAEN, Université de Caen, 6 boulevard du Maréchal Juin, 14050 Caen, France

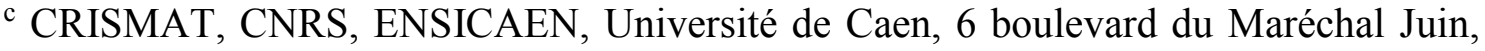
14050 Caen, France

\begin{abstract}
Antimicrobial resistance is a global threat with catastrophic forecasts in terms of human and economic losses. The so-called ESKAPE pathogens (Enterococcus species, Staphylococcus aureus, Klebsiella pneumoniae, Acinetobacter baumanii, Pseudomonas aeruginosa and Enterobacter species) represent a range of species of particular concern because they cause many serious hospital infections, and can show resistance toward available commercial antibiotics. Copper-containing zeolite nanocrystals $(10-30 \mathrm{~nm})$ with FAU-type structure (Cu-FAU), in the form of stable colloidal suspensions, were prepared at high yield in the absence of organic templates and studied for their activity against ESKAPE microorganisms. The materials were active against all six ESKAPE species. The survival of Staphylococcus aureus, Klebsiella pneumoniae and Pseudomonas aeruginosa on stainless-steel coupons after direct treatment with the $\mathrm{Cu}-$ FAU zeolite suspensions was determined quantitatively. Complete decontamination (5-
\end{abstract}


$\log$ reduction in bacterial counts) was achieved within 20 minutes for $P$. aeruginosa, and within 10 minutes for the $K$. pneumoniae and $S$. aureus. This result is significant, particularly for sanitization of surfaces in healthcare settings, with the potential to initiate a new direction of research to help address the global antimicrobial resistance threat.

Keywords: Nanozeolite, suspensions, copper, antibacterial, ESKAPE pathogens 


\section{Introduction}

Colloquially known as the ESKAPE pathogens, Enterococcus species, Staphylococcus aureus, Klebsiella pneumoniae, Acinetobacter baumanii, Pseudomonas aeruginosa and Enterobacter species have been identified by the Infectious Disease Society of America as the microorganisms most commonly causing infections that can be resistant to antimicrobial agents [1,2]. Antimicrobial resistance (AMR) is a naturally occurring evolution whereby microorganisms are capable of withstanding treatment by antimicrobial agents. It is estimated that about 700,000 people die each year due to drug resistant infections, with the potential for this number to rise to 10 million deaths a year by 2050 , succeeding the number of deaths by cancer, causing economic losses of 100 trillion USD [3]. In light of this global crisis, research into antimicrobial agents has begun to focus on ESKAPE pathogens as model targets. In this work, we use copper-containing zeolite nanocrystals for the first time as antimicrobial carriers against ESKAPE pathogens. Moreover, we employ zeolites in the form of stable colloidal suspensions for direct sanitization of surfaces.

Zeolites in the form of stable colloidal suspensions with a size of $100 \mathrm{~nm}$ or less were developed in the early 1990s [4]. The next 10-15 years saw a tremendous academic interest in nanozeolites sparked by the opportunities to gain insights into zeolite crystallization mechanisms and to prepare novel zeolite materials such as films and membranes [5]. However, the unsustainable synthesis of nanozeolites, characterized by very low yields and abundant use of structure-directing templates, gradually shifted research efforts towards developments of other materials, for instance hierarchical zeolites [6]. A breakthrough in nanozeolite research was reported in 2012 with the synthesis of ultrasmall EMT-type zeolite from a template-free system under mild 
conditions at a very high yield [7]. The approach used was recently applied to prepare nanosized FAU-type zeolites, and the advantages of using these nanosized zeolites in catalysis compared to a high quality commercial micron-sized zeolite were clearly demonstrated [8]. The development of green and industrially-friendly methods for synthesis of nanosized zeolites in the form of stable colloidal suspensions is now enabling their exploration in more conventional application areas such as microbiology.

Zeolites have been used in antimicrobial applications after ion-exchange of an antimicrobial metal, most often silver [9-16]. Owing to their microporosity and high surface areas, silver-exchanged zeolites slowly release silver ions in solution, creating a material with long-term antimicrobial activity [17]. Silver-exchanged zeolites in the form of zeolite supported films or additives to polymers and polymer coatings have also shown potential for different antimicrobial applications [18-20]. Although silver has demonstrated antimicrobial activity against a broad spectrum of microorganisms, concerns exist about the widespread use of silver nanoparticles in household products, cosmetics and textiles, leading to an observed accumulation of silver in the environment [21]. In addition, silver can easily be reduced to metallic silver, which requires special treatment for use and could affect the activity of materials. Copper is a cheaper and more stable metal than silver. It has attracted considerable research interest in recent years, particularly in hospital settings [22,23]. Copper has also been hosted within zeolites for potential environmental applications [24-26]. Although the mechanism of the antibacterial effect of copper cations is still not well understood, it has been suggested that the metal cations enter the cells and damage the bacterial cell membranes through the formation of reactive oxygen species [27]. 
The aims of the present study were to evaluate the potential of using $\mathrm{Cu}$ nanozeolite suspensions against ESKAPE pathogens and to develop relevant microbiology methods to test the antibacterial activity of $\mathrm{Cu}$-nanozeolites. EMT- and FAU-type zeolites are the only zeolites that have been prepared in the form of stable colloidal suspensions without the use of organic templates [7,8]. FAU-type zeolite was selected in this work because of its more extensive use in antibacterial studies. The activity of ultrasmall 10-30 nm Cu-containing FAU-type zeolites (Cu-FAU) materials against six ESKAPE microorganisms, Enterococcus faecalis (E. faecalis), Staphylococcus aureus (S. aureus), Klebsiella pneumoniae (K. pneumoniae), Acinetobacter baumanii (A. baumannii), Pseudomonas aeruginosa (P. aeruginosa) and Enterobacter cloacae (E. cloacae), and a standard reference microorganism, Escherichia coli (E. coli), was studied semi-quantitatively. Following this, the zeolite suspensions were applied to stainless steel coupons fouled with bacteria to quantitatively establish the survival of $S$. aureus, $K$. pneumoniae and $P$. aeruginosa and to assess the surface decontamination potential of $\mathrm{Cu}$ nanozeolites.

\section{Experimental}

\subsection{Synthesis of $\mathrm{Cu}-\mathrm{FAU}$}

The template-free, nanosized FAU zeolite was prepared from a precursor mixture with the molar composition: $9 \mathrm{Na}_{2} \mathrm{O}: 1.1 \mathrm{Al}_{2} \mathrm{O}_{3}: 10 \mathrm{SiO}_{2}: 77 \mathrm{H}_{2} \mathrm{O}$ [8]. Firstly, solutions A and B were prepared as follows: Solution A was made by dissolving $2.5 \mathrm{~g}$ of sodium hydroxide (NaOH, $97 \%$, Sigma-Aldrich) in $3.0 \mathrm{~g}$ of distilled water, followed by gradual addition of $0.297 \mathrm{~g}$ aluminum powder (325 mesh, $99.5 \%$, Alfa Aesar). The mixture was stirred until a clear solution was obtained. Solution B was prepared by dissolving $1.1 \mathrm{~g}$ of $\mathrm{NaOH}$ in $1.0 \mathrm{~g}$ of distilled $\mathrm{H}_{2} \mathrm{O}$, followed by the addition of $10 \mathrm{~g}$ colloidal silica (Ludox- 
HS 30, $30 \mathrm{wt} \% \mathrm{SiO}_{2}$, Aldrich). The resultant turbid suspension was placed in an oven at $100{ }^{\circ} \mathrm{C}$ for $5 \mathrm{~min}$ in order to obtain a clear suspension.

Solution A was added dropwise to solution B under vigorous stirring; solution B was kept in ice during the addition. The resulting clear suspension was aged with stirring at room temperature for $24 \mathrm{~h}$ followed by dehydration by freeze-drying to adjust the desired water content. The hydrothermal treatment was performed at $50{ }^{\circ} \mathrm{C}$ for $26 \mathrm{~h}$. The crystalline product was diluted with hot distilled water $\left(80{ }^{\circ} \mathrm{C}\right)$ and purified by centrifugation (20000 rpm, $25 \mathrm{~min}$ ) and redispersion in distilled $\mathrm{H}_{2} \mathrm{O}$. Part of this nanosized Na-FAU suspension was freeze-dried for further characterization and the rest was kept as suspension for post-synthetic $\mathrm{Cu}$ ion-exchange.

The Na-FAU zeolite suspension was ion-exchanged with copper (II) nitrate $(\mathrm{Cu}$ $\left.\left(\mathrm{NO}_{3}\right)_{2}\right)$ to prepare $\mathrm{Cu}-\mathrm{FAU}$. The ion-exchange procedure was carried out as follows: 50 $\mathrm{mL}$ of $\mathrm{Cu}\left(\mathrm{NO}_{3}\right)_{2} .9 \mathrm{H}_{2} \mathrm{O}(99-104 \%$, Sigma-Aldrich) with a concentration of $0.008 \mathrm{M}$ was added to $10 \mathrm{~mL}$ of Na-FAU zeolite suspension (3.6 wt \% zeolite) and vigorously stirred at room temperature for $1 \mathrm{~h}$. This was followed by purification via centrifugation and redispersion in distilled water. The purification process was repeated twice under the same conditions. After the second ion-exchange, the final samples were washed twice with distilled water by centrifugation at $20000 \mathrm{rpm}$ for $25 \mathrm{~min}$, and re-dispersed in distilled $\mathrm{H}_{2} \mathrm{O}$. The final $\mathrm{pH}$ of the $\mathrm{Cu}-\mathrm{FAU}$ suspensions was 7.0.

\subsection{Characterization methods}

The crystal structure of the parent Na-FAU and the ion-exchanged zeolite $\mathrm{Cu}$ FAU was determined using a PANalytical X'Pert Pro diffractometer with $\mathrm{Cu} \mathrm{K} \alpha$. The morphology of the synthesized materials was studied by scanning electron microscope (SEM) taken on MIRA-LMH (TESCAN) supplied with a field emission gun. Further, the 
samples were studied by transmission electron microscopy (TEM). TEM analysis was carried out using a $200 \mathrm{kV}$ JEOL 2010 FEG electron microscope. Energy dispersive spectroscopy coupled with the TEM instrument was used to study the Cu-FAU sample. The stability of the zeolite suspensions at $\mathrm{pH}=7.0$ before and after the ion-exchange was measured by zeta potential using a Malvern Zetasizer Nano instrument. The elemental analysis of the samples was determined by inductively coupled plasma-atomic emission spectroscopy (ICP-AES) using an OPTIMA 4300 DV (PerkinElmer) instrument.

\subsection{Microorganisms}

Pseudomonas aeruginosa NCTC 6749, Staphylococcus aureus NCTC 6571, Enterobacter cloacae NCTC 10005, Acinetobacter baumannii NCTC 12156, Klebsiella pneumoniae NCTC 9633 and Enterococcus faecalis NCTC 775 (ESKAPE pathogens) as well as Escherichia coli ATCC 8739 (reference microorganism) were maintained on Nutrient Agar (Oxoid, Basingstoke) at $5{ }^{\circ} \mathrm{C}$ and inoculated into $100 \mathrm{~mL}$ Nutrient Broth (Oxoid). For each experiment and each culture, 2-4 colonies were inoculated into $100 \mathrm{~mL}$ Nutrient Broth (Oxoid) and grown overnight $(22 \pm 1 \mathrm{~h})$ at $37^{\circ} \mathrm{C}$ with agitation $(180 \mathrm{rpm})$. Cells were harvested by centrifugation (3600 rpm, $10 \mathrm{~min}$ ) and washed once in $0.85 \%$ $\mathrm{NaCl}$ (Oxoid), resuspended to optical density 1.0 at $540 \mathrm{~nm}$ (Jenway 6305 Spectrophotometer, UK) resulting in a suspension concentration of $3.24 \pm 0.14 \times 10^{9} \mathrm{CFU}$ $\mathrm{mL}^{-1}$.

\subsection{Semi-quantitative testing for microbial kill}

E. faecalis suspension $(25 \mu \mathrm{L})$ was added to $5 \mathrm{~mL}$ of $2 \mathrm{mg} \mathrm{mL}^{-1}$ of $\mathrm{Cu}-\mathrm{FAU}$, whilst $25 \mu \mathrm{L}$ of the remaining strains were added to $5 \mathrm{~mL}$ of $1 \mathrm{mg} \mathrm{mL}^{-1}$ of Cu-FAU. The $\mathrm{Cu}$-free FAU was used for control experiments. The samples were incubated at $37^{\circ} \mathrm{C}$ in a rotary shaker incubator set to $150 \mathrm{rpm}$ and a $20 \mu \mathrm{L}$ sample was taken every 40 min over 
a period from 0-280 min for E. faecalis and every 20 min over a period of 0-140 min for all other microorganisms. Each $20 \mu \mathrm{L}$ sample was pipetted onto one segment of a thioglycollate agar plate and a nutrient agar plate (Oxoid) which had been divided into eight segments. The thioglycollate agar was prepared by mixing thioglycollate broth (Oxoid) with 1.2\% wt technical agar (Oxoid). All tests were carried out in duplicate and the experiment was repeated. Plates were incubated at $37^{\circ} \mathrm{C}$ overnight and examined for the amount of bacterial growth following exposure to the Cu-FAU samples. A decrease in colony numbers was apparent as time progressed but this was not scored. Kill was noted as the total absence of colonies.

\subsection{Survival of microorganisms on stainless-steel (SS) in the presence of Cu-FAU}

Following semi-quantitative analysis for microbial kill, $P$. aeruginosa, $K$. pneumoniae and S. aureus were selected for assessing survival on SS in the presence of Cu-FAU. SS (316 2B) 2x2 cm coupons and polyethylene (PE) sheets (SLS, Nottingham, UK) cut to $2 \times 2 \mathrm{~cm}$ were soaked in ethanol for 30 minutes, washed with sterile distilled water and left to dry for 90 minutes in a class two cabinet (BH-EN 2003, Faster, Cornaredo). $50 \mu \mathrm{L}$ of bacterial culture was placed onto a SS coupon, spread using the end of a sterile pipette tip, and left to dry on the surface for 90 minutes in a class two cabinet. Once dry, $50 \mu \mathrm{L}$ of $\mathrm{Cu}-\mathrm{FAU}$ was pipetted onto the $\mathrm{SS}$, and a $2 \times 2 \mathrm{~cm}$ piece of polyethylene was placed on top of the inoculum to ensure the liquid was evenly distributed over the SS coupon. At the appropriate sample time, the SS coupon with PE sheet was dropped into $10 \mathrm{~mL}$ of neutralizing buffer (15 g Tween80 (Sigma- Aldrich, Dorset) and $30 \mathrm{~g}$ Soya Lecithin (Optima Healthcare Lecithin, Holland and Barrett, UK) dissolved in 1 L of distilled water) [28]. The role of this neutralizing buffer was to halt the antibacterial action of copper at the appropriate time point. Neutralizing buffer was then vortex-mixed for 30 
sec to remove the polyethylene from SS and remove any cells from the surface into the liquid. The bacterial suspension was then diluted 10 -fold $(1 \mathrm{~mL}$ into $9 \mathrm{~mL}$ of sterile physiological saline), seven times, to a concentration of $10^{-7}$ of the original suspension. $100 \mu \mathrm{L}$ of each dilution was pipetted out in duplicate on nutrient agar and spread evenly across the agar surface. Plates were incubated for $18 \mathrm{~h}$ at $37^{\circ} \mathrm{C}$, after which the number

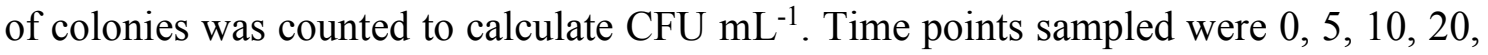
40 and 60 minutes. All samples were duplicated and the experiment was repeated.

\section{Results and discussion}

FAU-type zeolite was prepared in the absence of organic template as described previously [8]. TEM images indicated that the sample was fully crystalline with crystallites in the size range 10-30 $\mathrm{nm}$ (Fig. 1a). The zeolite nanocrystals are well-shaped and most of them exhibit the typical octahedral morphology with well-developed faces. Depending on crystals orientation on the grid, they appear as bi-pyramidal, isometric or even plate-like. $\mathrm{Cu}$ was introduced into the FAU-type zeolite by an ion-exchange approach. Elemental analysis indicated that $75 \%$ of $\mathrm{Na}$ was exchanged resulting in 11 wt.\% $\mathrm{Cu}$ content in the $\mathrm{Cu}-\mathrm{FAU}$ sample (Table $\mathrm{S} 1$, Supporting information). The $\mathrm{Cu}-$ exchange did not affect the $\mathrm{Si} / \mathrm{Al}$ ratio, which was 1.2 for both Na-FAU and $\mathrm{Cu}-\mathrm{FAU}$ zeolites. TEM showed that the ion-exchange did not affect the zeolite crystallinity, crystal size or shape (Fig. 1b). This conclusion was further confirmed by XRD and SEM analysis. XRD patterns of the Na-FAU and Cu-FAU were typical of the FAU-type zeolite with similar intensity of the XRD peaks indicating that the zeolite structure was preserved during the $\mathrm{Cu}$ ion-exchange (Fig. 1c). The SEM images showed that the zeolite crystallites were well-shaped with a narrow particle size distribution in both Na-FAU and Cu-FAU samples (Fig. S1, Supporting Information). 
The $\mathrm{Cu}$ content in the zeolite crystals was determined based on EDS (Fig. 2); with the copper signal coming only from the zeolite particles since Ni-grids were used to prepare the samples. Under prolonged exposure of the crystals to the e-beam (1 minute), the $\mathrm{Cu}$ nanoparticles were reduced and aggregated as shown in the Supporting Information, Fig. S2.

The colloidal stability of the prepared zeolite suspensions is of paramount importance for the application of the materials to treat surfaces. The stability of the zeolite samples was studied using zeta potential measurements (Fig. 3). Usually, a zeta potential threshold value of $\pm 30 \mathrm{mV}$ is used to measure stability; values above this threshold value indicate colloidal stability. The zeta potential of the zeolite suspensions varied between $41.5 \mathrm{mV}$ and $-45.6 \mathrm{mV}$ for $\mathrm{Na}-\mathrm{FAU}$ and $\mathrm{Cu}-\mathrm{FAU}$, respectively, indicating that both suspensions were colloidally stable. The colloidal stability of the samples can be seen clearly from the digital images in Fig. 3, inset. In addition, the samples did not aggregate upon storage for at least 3 months. After $\mathrm{Cu}$ ion-exchange, the suspension was a lightbluish color. DLS results (Fig. 3b) indicated a monomodal particle size distribution, which was similar for the Na-FAU and Cu-FAU samples.

The antibacterial properties of the Cu-FAU suspensions were firstly studied using our previously developed semi-quantitative method with $E$. coli as a reference microorganism [14]. The Cu-free samples (Na-FAU) were used as controls throughout this work and they showed no antibacterial activity in all experiments. In our previous work, we used Ag-loaded EMT zeolites with zeolite concentrations of $0.5 \mathrm{mg} \mathrm{mL}^{-1}$ [14]. Killing times were achieved within less than 10 minutes depending on the Ag content and the $\mathrm{Ag}$ form, metallic or ionic. Initial experiments using $\mathrm{Cu}-\mathrm{FAU}$ with zeolite concentrations of $0.5 \mathrm{mg} \mathrm{mL}^{-1}$ did not kill $E$. coli quickly enough (within 15 minutes) to 
provide a viable testing method, for which reason the concentration in all further experiments was doubled to $1 \mathrm{mg} \mathrm{mL} \mathrm{m}^{-1}$. Similarly to our previous results, the use of thioglycollate agar as a growth medium was important to halt the antibacterial action of $\mathrm{Cu}$ at the corresponding sampling time (Fig. S3, Supporting information). E. coli was killed within 35 min when thioglycollate agar was used and within 20 min using nutrient agar. These experiments indicated that the $\mathrm{Cu}$-exchanged ultrasmall zeolites had lower antibacterial activity compared to Ag-exchanged ultrasmall zeolites. We have reported similar trends for FAU-type zeolites prepared from fly ash after ion-exchange with Ag and $\mathrm{Cu}[26]$.

After optimization of the semi-quantitative tests in terms of zeolite concentrations using the reference $E$. coli, experiments continued using ESKAPE microorganisms. Typical images of the thioglycollate plates after incubation are shown in Fig. 4 with the average killing times summarized in the accompanying histogram. All experiments were performed in duplicates and repeated. Also, experiments were performed with $\mathrm{Cu}-\mathrm{FAU}$ suspensions from different batches to test reproducibility; similar results were obtained when samples from different batches were used. E. faecalis showed increased time to kill compared to the other ESKAPE microorganisms. Such increased time to kill E. faecalis compared to E. coli and P. aeruginosa was also observed in our previous work using Agexchanged zeolite prepared from fly ash [26]. Doubling the $\mathrm{Cu}-\mathrm{FAU}$ concentration to 2 $\mathrm{mg} \mathrm{mL}^{-1}$ and increasing the sampling time to $40 \mathrm{~min}$ indicated that $\mathrm{Cu}-\mathrm{FAU}$ reduced the E. faecalis viable cells and had the potential to kill this microorganism as well, although to a less extent compared to the other ESKAPE microorganisms (Fig. 4).

After establishing the potential of $\mathrm{Cu}-\mathrm{FAU}$ colloidal suspensions to deactivate ESKAPE microorganisms, quantitative tests were designed to determine the survival of 
selected microorganisms on stainless steel coupons. The introduction of copper alloy surfaces in intensive care units has been shown to decrease rates of healthcare-acquired infections [29]. However, the replacement of surfaces is not always appropriate / possible and deactivation of fouled surfaces by simple spraying, which is applicable to all types of surfaces, seems a much more elegant approach. Stainless steel has been selected in this study as one of the most relevant surfaces in healthcare settings. Based on the semiquantitative tests (Fig. 4), three key ESKAPE microorganisms were selected to study the capacity of $\mathrm{Cu}-\mathrm{FAU}$ to act as a surface decontamination agent, namely $K$. pneumoniae (shortest killing time), P. aeruginosa (middle killing time) and S. aureus (longest killing time amongst the microorganisms that died). In these experiments, bacterial cultures (50 $\mu \mathrm{L})$ were inoculated and left to dry on stainless steel coupons. Coupons were then treated with $50 \mu \mathrm{L}$ of $\mathrm{Cu}$-FAU. At the appropriate sample time, $0,5,10,20,40$ or 60 minutes, the coupons were dropped into a neutralizing buffer to halt the activity of $\mathrm{Cu}$ and the remaining viable cells on the surface were determined by the serial dilution method [28]. Complete kill was achieved within 20 minutes for P. aeruginosa, and within 10 minutes for the K. pneumoniae and $S$. aureus (Fig. 5). Bacterial cells survived on the surfaces when treated with Na-FAU. These results indicate that $\mathrm{Cu}$-FAU suspensions can be used for surface treatment to deactivate ESKAPE microorganisms.

The well-known antimicrobial properties of copper could similarly be demonstrated using a copper salt solution. However, there is little value in comparing such data with our zeolite work, since the zeolite can be immobilized in a solid matrix, generating a sustained release of copper ions over time, rather than an uncontrolled loss of ions. In addition, the zeolite provides an insoluble carrier for the copper ions, enabling containment and improved control of copper ion concentration in a flowing system, 
whereas copper salts would be immediately lost. Further, the use of $\mathrm{Cu}$-zeolites rather than copper solutions to decontaminate surfaces would minimize corrosion risks caused by the latter.

The present work provides scope for research in several directions. For the microorganisms studied, future work comparing survival over different time points up to $10 \mathrm{~min}$ (20 min for P. aeruginosa) to enable better comparison is suggested. Further work to determine the 'killing lifetime' of Cu-FAU suspensions sprayed onto surfaces and continuation of tests in hospital settings will be valuable based on the research reported here. The antimicrobial potential of $\mathrm{Cu}$-nanozeolites of different zeolite structures as well as optimization of the $\mathrm{Cu}$ loading are also worth studying.

\section{Conclusions}

Ultrasmall $\mathrm{Cu}$-FAU zeolite crystals were prepared in the form of stable colloidal suspensions. The Cu-FAU zeolites showed activity against $E$. coli and all six ESKAPE microorganisms, as determined by a semi-quantitative drop test. The survival of three selected ESKAPE microorganisms on stainless-steel surfaces treated with $\mathrm{Cu}-\mathrm{FAU}$ was studied quantitatively. None of the three microorganisms survived the $\mathrm{Cu}-\mathrm{FAU}$ treatment. This result is significant considering the urgent need for new antimicrobial drugs, especially drugs that are effective against Gram-negative pathogens such as Pseudomonas aeruginosa, Klebsiella pneumoniae and Acinetobacter baumanii [30]. The use of CuFAU colloidal suspensions to inactivate ESKAPE pathogens on surfaces, particularly in healthcare settings, could lead to the significant reduction of bacterial infections, thus reducing the need for antibiotics treatment.

\section{Acknowledgements}


This study was supported by the European Union-Fonds Européen de Développement Régional (FEDER).

\section{Appendix A. Supplementary data}

Supplementary data associated with this article can be found, in the online version, at

\section{References}

[1] H. W. Boucher, G. H. Talbot, J. S. Bradley, J. E. Edwards, D. Gilbert, L. B. Rice, M. Scheld, B. Spellberg, J. Bartlett, Clin. Infect. Dis. 48 (2009) 1-12.

[2] J. N. Pendleton, S. P. Gorman, B. F. Gilmore, Expert Rev. Anti Infect. Ther. 11 (2013) 297-308.

[3] J. O'Neill (Chair), The Review on Antimicrobial Resistance, Wellcome Trust, London, 2006.

[4] B. J. Schoeman, J. Sterte, J.-A. Otterstedt, J. Chem. Soc., Chem. Commun. (1993) 994-995.

[5] S. Mintova, M. Jaber and V. Valtchev, Chem. Soc. Rev. 44 (2015) 7207-7233.

[6] W. Schwieger, A. G. Machoke, T. Weissenberger, A. Inayat, T. Selvam, M. Klumpp, A. Inayat, Chem. Soc. Rev., 2016, 45, 3353.

[7] E.-P. Ng, D. Daniel Chateigner, T. Bein, V. Valtchev, S. Mintova, Science 335 (2012) $70-73$.

[8] H. Awala, J.-P. Gilson, R. Retoux, P. Boullay, J.-M. Goupil, V. Valtchev, S. Mintova, Nat. Mater. 14 (2015) 447-451.

[9] P. Lalueza, M. Monzón, M. Arruebo, J. Santamaria, Chem. Commun. 47 (2011) 680682.

[10] L. Ferreira, A. M. Fonseca, G. Botelho, C. Almeida-Aguiar, I. C. Neves, Microporous Mesoporous Mater. 160 (2012) 126-132. 
[11] P. Saint-Cricq, Y. Kamimura, K. Itabashi, A. Sugawara-Narutaki, A. Shimojima, T. Okubo, Eur. J. Inorg. Chem. (2012) 3398-3402.

[12] C. Carolina, B. J. Carlos, B. M. L. Zapata, Z. J. Manuel, Microporous Mesoporous Mater. 188 (2014) 118-125.

[13] Y. Zhou, Y. Deng, P. He, F. Dong, Y. Xia, Y. He, RSC Adv. 4 (2014) 5283-5288.

[14] B. Dong, S. Belkhair, M. Zaarour, L. Fisher, J. Verran, L. Tosheva, R. Retoux, J.P. Gilson, S. Mintova, Nanoscale 6 (2014) 10859-10864.

[15] S. A. Hanim, N. A. N. N. Malek, Z. Ibrahim, Appl. Surf. Sci. 360 (2016) 121-130.

[16] V. V. Singh, B. Jurado-Sánchez, S. Sattayasamitsathit, J. Orozco, J. Li, M. Galarnyk, Y. Fedorak, J. Wang, Adv. Funct. Mater. 25 (2015) 2147-2155.

[17] Y. Matsumura, K. Yoshikata, S. Kunisaki, T. Tsuchido, Appl. Environ. Microbiol. 69 (2003) 4278-4281.

[18] K. A. Rieger, H. J. Cho, H. F. Yeung, W. Fan, J. D. Schiffman, ACS Appl. Mater. Interfaces 8 (2016) 3032-3040.

[19] S. Belkhair, M. Kinninmonth, L. Fisher, B. Gasharova, C. M. Liauw, J. Verran, B. Mihailova, L. Tosheva, RSC Adv. 5 (2015) 40932-40939.

[20] M. M. Cowan, K. Z. Abshire, S. L. Houk, S. M. Evans, J. Ind. Microbiol. Biotechnol. 30 (2003) 102-106.

[21] C. Marambio-Jones, E. M. V. Hoek, J. Nanopart. Res. 12 (2012) 1531-1551.

[22] G. Grass, C. Rensing, M. Solioz, Appl. Env. Microbiol. 77 (2011) 1541-1547.

[23] M. Vincent, P. Hartemann, M. Engels-Deutsch, Int. J. Hyg. Environ. Health. 219 (2016) 585-591.

[24] R. Tekin, N. Bac, Microporous Mesoporous Mater. 234 (2016) 55-60. 
[25] J. Behin, A. Shahryarifar, H. Kazemian, Chem. Eng. Technol. 39 (2016) 23892403.

[26] L. Tosheva, A. Brockbank, B. Mihailova, J. Sutula, J. Ludwig, H. Potgieter, J. Verran, J. Mater. Chem. 22 (2012) 16897-16905.

[27] C. Ning, X. Wang, L. Li, Y. Zhu, M. Li, P. Yu, L. Zhou, Z. Zhou, J. Chen, G. Tan, Y. Zhang, Y. Wang, C. Mao, Chem. Res. Tox. 28 (2015) 1815-1822.

[28] L. Fisher, S. Ostovapour, P. Kelly, K. A. Whitehead, K. Cooke, E. Storgårds, J. Verran, Biofouling 30 (2014) 911-919.

[29] C. D. Salgado, K. A. Sepkowitz, J. F. John, J. Robert Cantey, H. H. Attaway, K. D. Freeman, P. A. Sharpe, H. T. Michels, M. G. Schmidt, Infect. Control Hosp. Epidemiol. 34 (2013) 479-486.

[30] R. Tommasi, D. G. Brown, G. K. Walkup, J. I. Manchester, A. A. Miller, Nat. Rev. 14 (2015) 529-542. 


\section{Figure captions}

Figure 1. TEM images of (a) Na-FAU and (b) Cu-FAU (Scale bar $=20 \mathrm{~nm}$ ), and (c) XRD patterns of $\mathrm{Na}-\mathrm{FAU}$ and $\mathrm{Cu}-\mathrm{FAU}$.

Figure 2. EDX spectra of (a) Na-FAU and (b) Cu-FAU samples measured on Ni grids. The chemical composition of the samples retrieved from the EDX is shown in Table S2.

Figure 3. (a) Zeta potential and (b) DLS curves of Na-FAU (solid line) and Cu-FAU (dotted line) samples; Inset: digital images of the Na-FAU (white) and Cu-FAU (blue) zeolite suspensions.

Figure 4. Left: Spot inoculation of ESKAPE microorganisms following exposure to the $\mathrm{Cu}-\mathrm{FAU}$ suspension. Each drawn segment on the plates above corresponds to 20 minutes sampling time (40 minutes for E. faecalis); total sampling time 0-140 minutes (0-280 minutes for E. faecalis). Microorganisms (clockwise): K. pneumoniae, E. cloacae, $P$. aeruginosa, A. baumannii, S. aureus and E. faecalis. Right: Summary of the average killing times determined in the semi-quantitative tests.

Figure 5. Survival of bacterial strains on stainless steel coupons: (a) S. aureus, (b) $K$. pneumoniae, and (c) P. aeruginosa. 

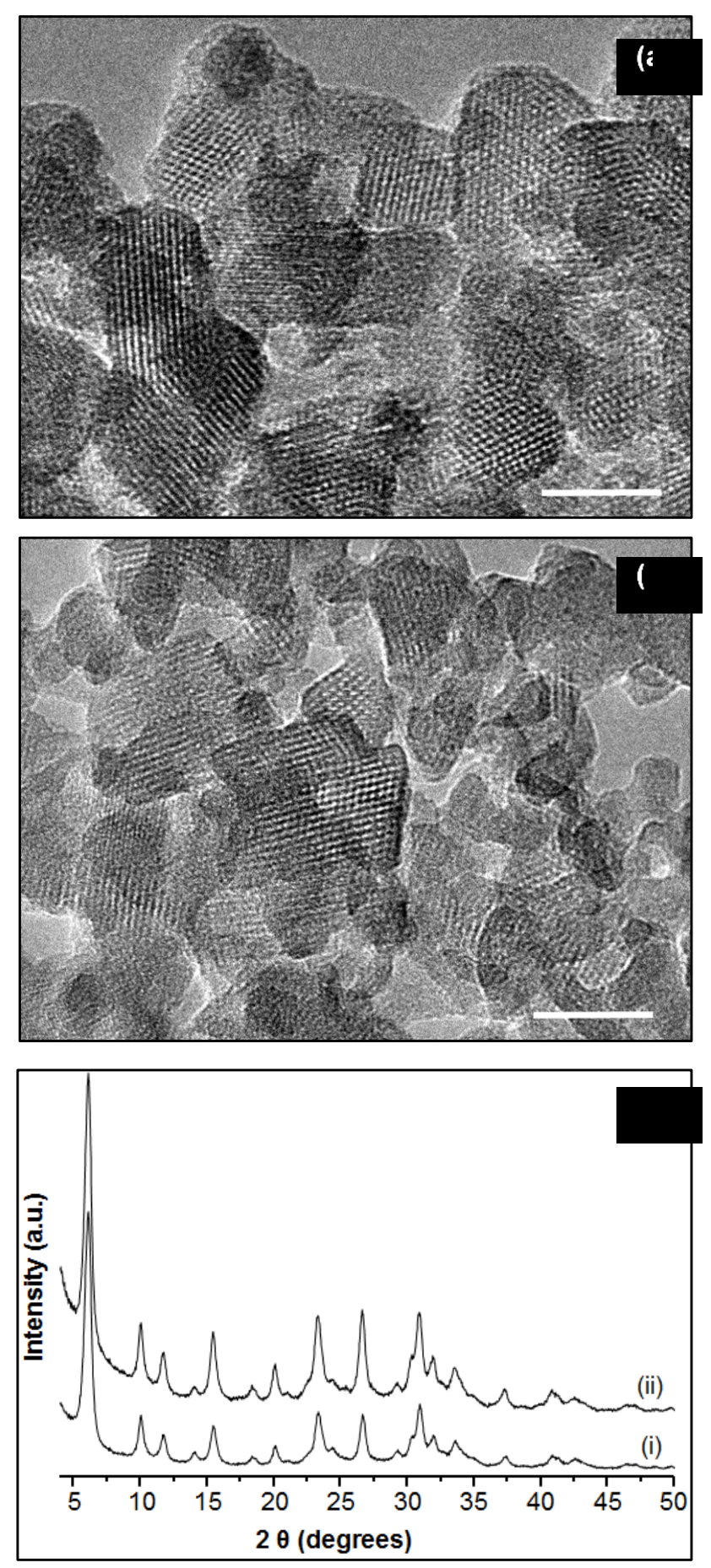

Figure 1 

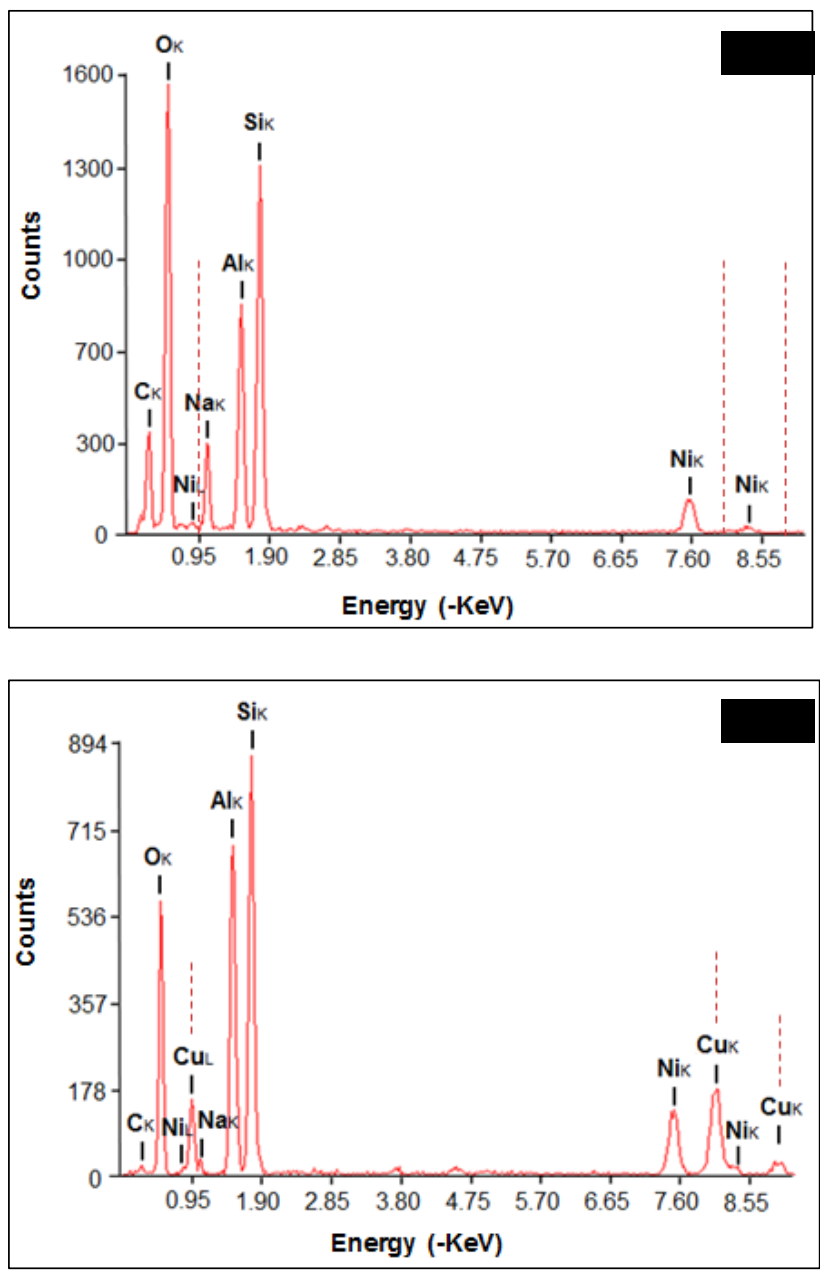

Figure 2 

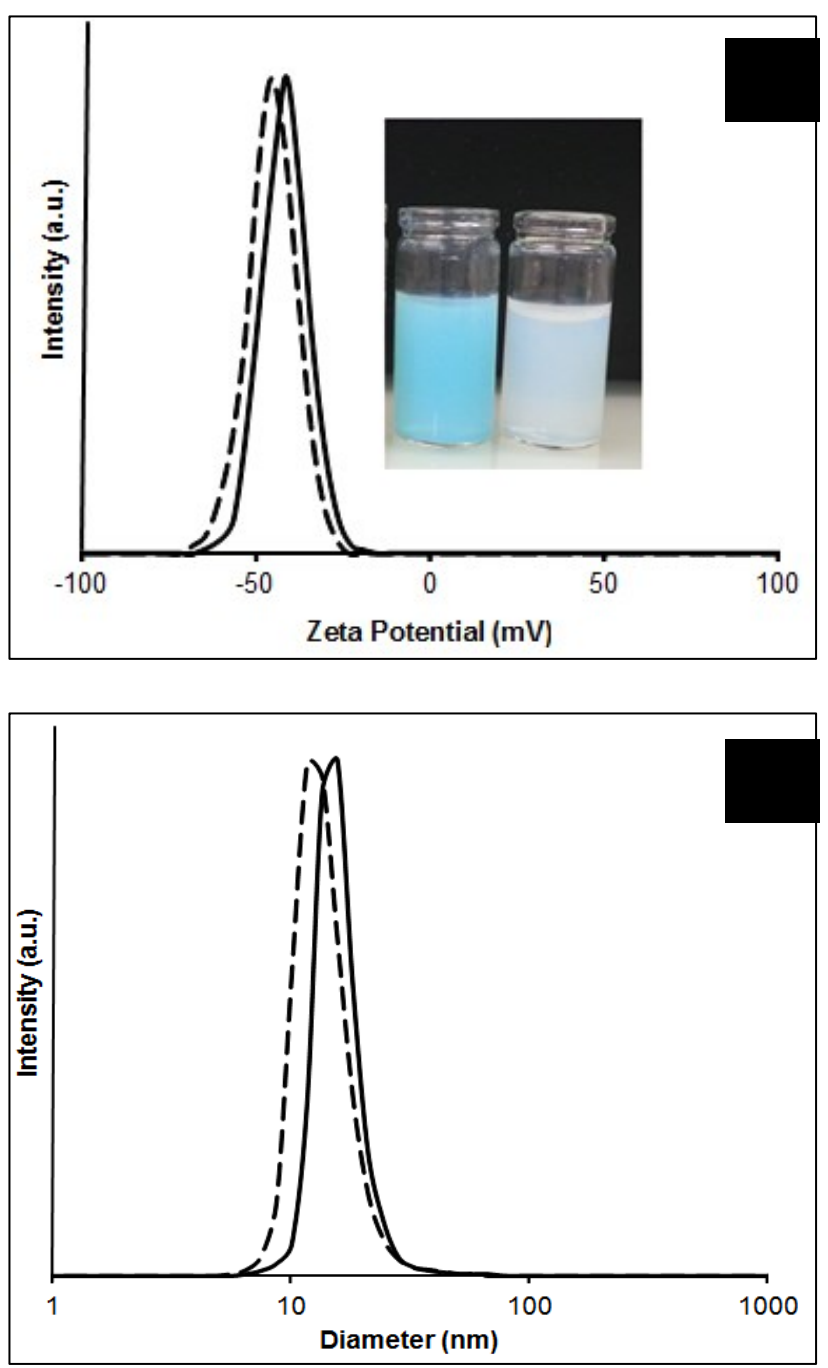

Figure 3 

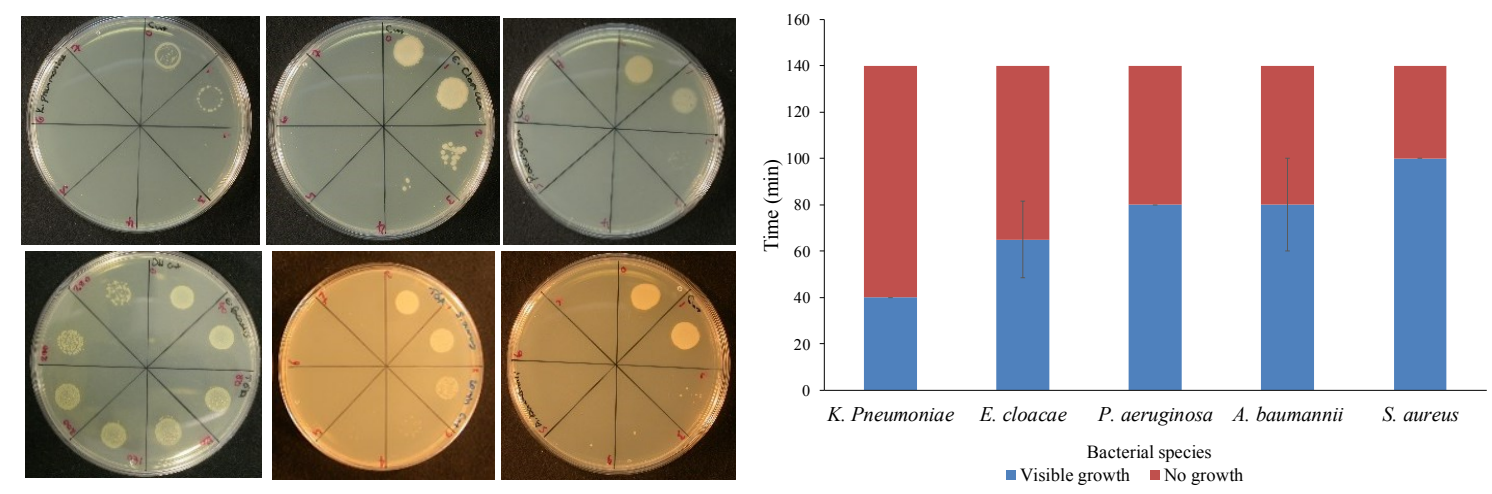

Figure 4 


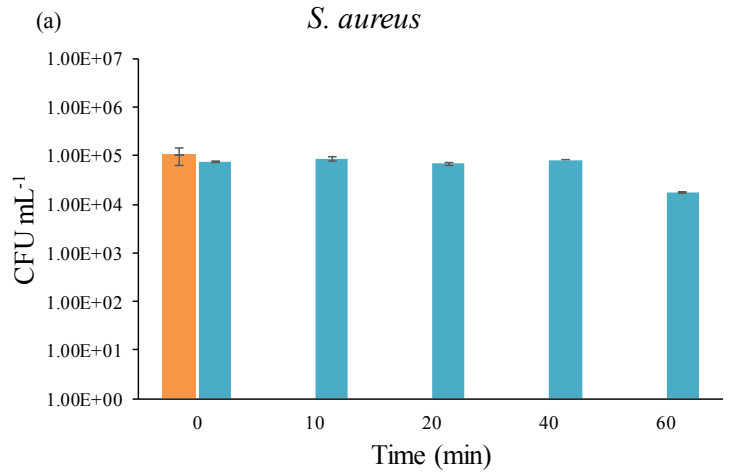

In the presence of $\mathrm{Cu}-\mathrm{FAU}$ In the absence of $\mathrm{Cu}-\mathrm{FAU}$

(b)

K. pneumoniae

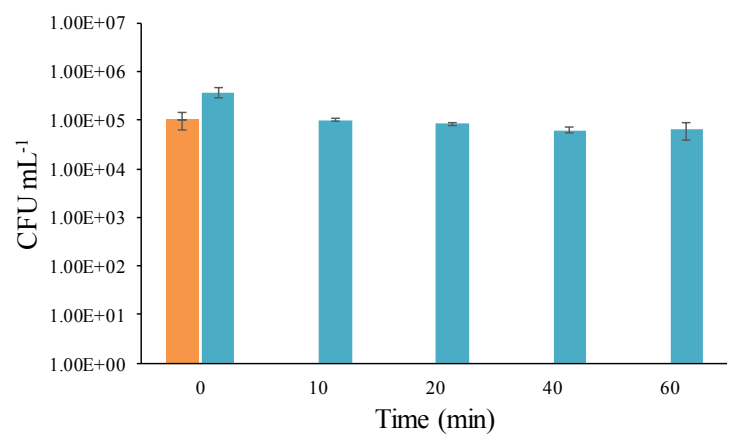

In the presence of $\mathrm{Cu}-\mathrm{FAU} \quad$ In the absence of $\mathrm{Cu}-\mathrm{FAU}$

(c)

P. aeruginosa

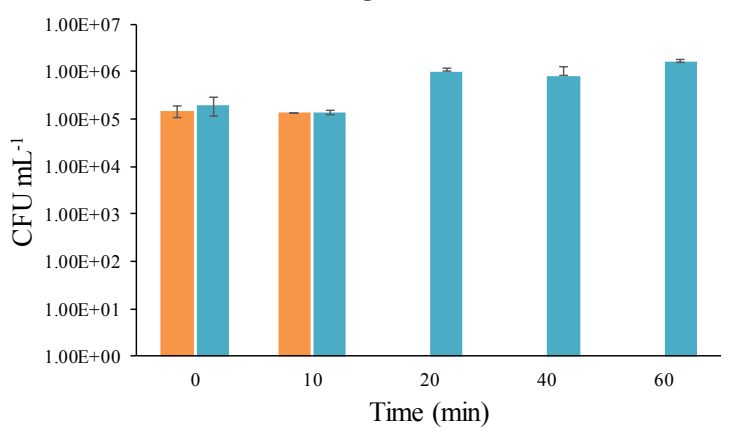

In the presence of $\mathrm{Cu}-\mathrm{FAU} \quad \mathrm{In}$ the absence of $\mathrm{Cu}-\mathrm{FAU}$

Figure 5 